\title{
Design of Network Educating Information System Based on Use Cases Driven
}

\author{
Shenwei Wang ${ }^{1} \&$ Min Guo ${ }^{2}$ \\ ${ }^{1}$ College of Electronic and Control Engineering, Beijing University of Technology, Beijing 100124, \\ China
}

${ }^{2}$ Engineering Research Center of Digital Community, Ministry of Education, Beijing 100124, China

Keywords: use cases driven; information system; network education.

\begin{abstract}
Based on the current situation about education and teaching, a set of network educating information system is designed, which concentrates on enhancing efficiency of education. The system realizes online educating and management, including series of functional models, such as education information management, course designing, online studying, and feedback of teaching results and so on. On the foundation of systematic division by target decomposition, UML modeling based on use cases driven is taken to process the system analysis and design the system with service-oriented architecture. The system has been developed and applied successfully by an educational organization in Beijing. In the process of network educating, the system provides multiple modes for teaching, and improving the efficiency of educational management.
\end{abstract}

\section{Introduction}

With development in society, there is a huge gap between the number of the talented people and the need of economic progress. Many colleges and universities are developing network education as a way for improving the studying environment, reforming the traditional teaching mode and adapting on development of web and information technology. With the development and maturity of web technique, people usually process teaching resources in high quality by web and information technology to make the resources spread widely on Internet. But there are many network educating systems that are just online textbooks which cannot assist students in studying. These systems not only have just single teaching mode, but also ignore interactive charter of web, as consequence of the phenomenon that the content that is what the students do not want to learn the content that the system provides.

In addition, during the phase of requirement analysis, it is easier to emerge mistakes without professional instruction because education is strongly professional filed. According to the theory offered by Leffingwell that over $40 \%$ problems occur when people cope with requirement analysis in the procedure of developing software, acquisition of accurate system requirement is also a key point of this paper. So the efforts of building a network educating information system to overcome these shortages mentioned above are extremely significant.

To sum up, the network educating information system has been completely designed. Depending on target decomposition, UML modeling based on use cases driven is used to design and analyze the system. By this way, the clear structure of system and the traceability between the requirement and design can be displayed. The tool of modeling and designing is Astah in the system.

\section{Requirement Analysis}

\section{1 system organization}

In order to organize the network educating system, which including many complex procedures such as teaching service, course designing, educational administration management and so on, target decomposition is used to divide the system. Firstly, confirmed by preliminary system investigation, the goal of the system comprises of improving efficiency of teaching management, enhancing quality of higher education, pushing open wider of higher education, and motivating the construction of learning society. By analyzing the goal of the system, it involves four aspects of network educating: 
education management, assisted instruction, learning knowledge, and sharing the resources. For the aspect of education management, the system is mainly utilized to manage the information of education. Aiming at safe and efficient management, the system complies with the standard of information system, so one of the sub targets is efficient management relevant information relying on information system. For the aspect of assisted instruction, flexible teaching mode is a critical point to promote the quality of education. Therefore, second sub objective is to enhance the quality of education by providing multiple educating modes. For the aspect of learning knowledge, it is very crucial that the system promptly notifies the both sides about the results of learning by feedback from the system, thus the third sub goal is to acquire promptly dynamic of studying by information technology. Finally, for the part of sharing the resources, owing to information exchange on Internet having many advantages such as interactive and low cost, sharing resources can be implemented by web. Consequently, the last sub target is to spread educational resources wildly by network model. The result of the target divided is shown in the picture 1 .

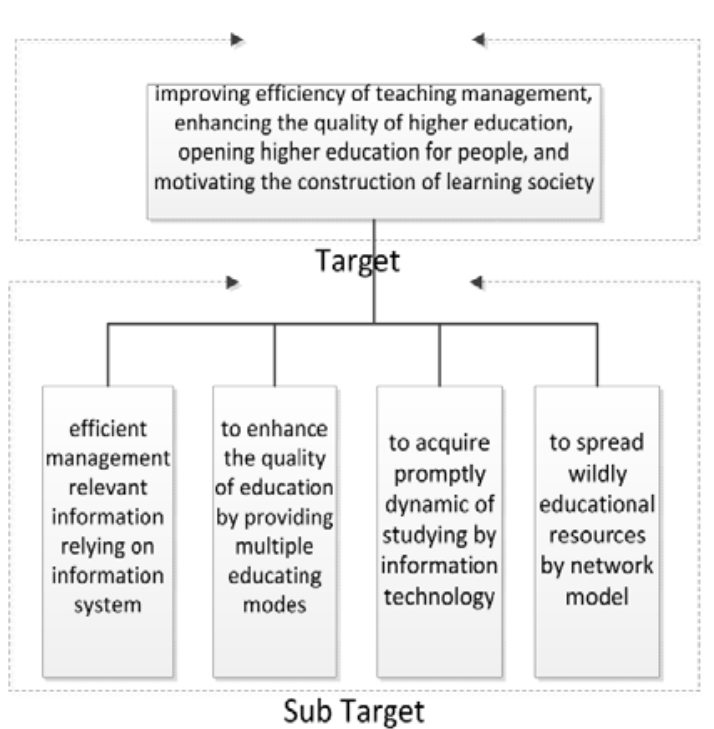

Figure 1: Target divided

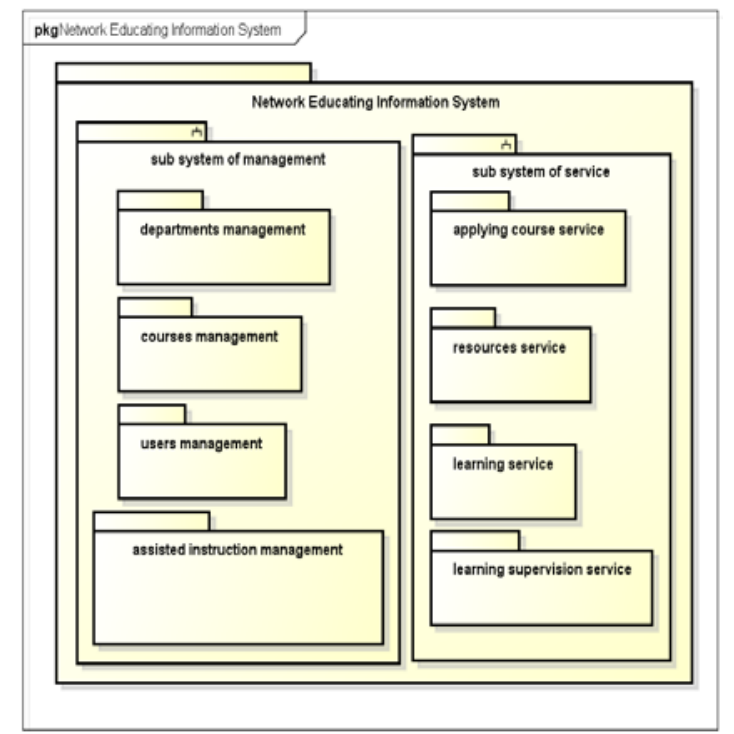

Figure 2: System organization

Based on the sub target of efficient management relevant information relying on information system, the system manages information containing the information of the department's structure, courses information and teachers and student's information, by analyzing information of colleges and universities, so there are three modules of departments management, courses management, and users management included in the system. On the basis of the second sub objective of enhancing educational quality by providing multiple educating modes, there are many kinds of educating modules and ways to compose, therefore the system needs to have the module of assisted instruction management to administrate these educating modules. In view of the third sub goal of prompt acquaintance of studying dynamic by information technology, it is necessary to collect the learning data and deliver the results of statistic to the teacher and the student, thus it also requires a module of learning supervision service in the system. On account of the last sub target of spreading educational resources wildly by Network model, the system provides a platform of sharing resources for users, consequently, it has a module of resources service. Owing to the system applied in colleges and universities, there are still two modules to provide including applying course service and learning service. In conclusion, the systematic organization can be built by above analysis, which includes in the part of sub system of management: department's management, courses management, user's management, assisted instruction management, and in the part of sub system of service: applying course service, learning service, learning supervision service, resources service. The result of the systematic organization is shown in the picture 2 .

\section{2 use case model}

On the foundation of systematic organization, the article discusses function requirements under a smaller system boundaries by the way of use case model. Because there are many characters belonging to use cases such as atomicity, encapsulation and observability(by observing the results of 
use case), from the perspective of black box to find function requirements in the system, Interactive sequences between specific actors and the system are encapsulated in use cases whose accuracy is validated by atomicity and observability. There are there kinds of actors that consist of administrator, teacher and student. Under the corresponding system boundary, the administrator can execute the functions which include maintaining department's structure, establishing or forbidding courses, registering or eliminating users. The student has behaviors consisting of applying for learning, completing educational activities such as debates, homework, quizzes. The actions of the teacher comprise of applying for establishing courses, applying for teaching, formulating arrangement of course, managing educational activities and checking educational statistic. Teachers with students can share the resources of education and participate in Q\&A of course. According to above analysis, the use case model is depicted in picture 3.

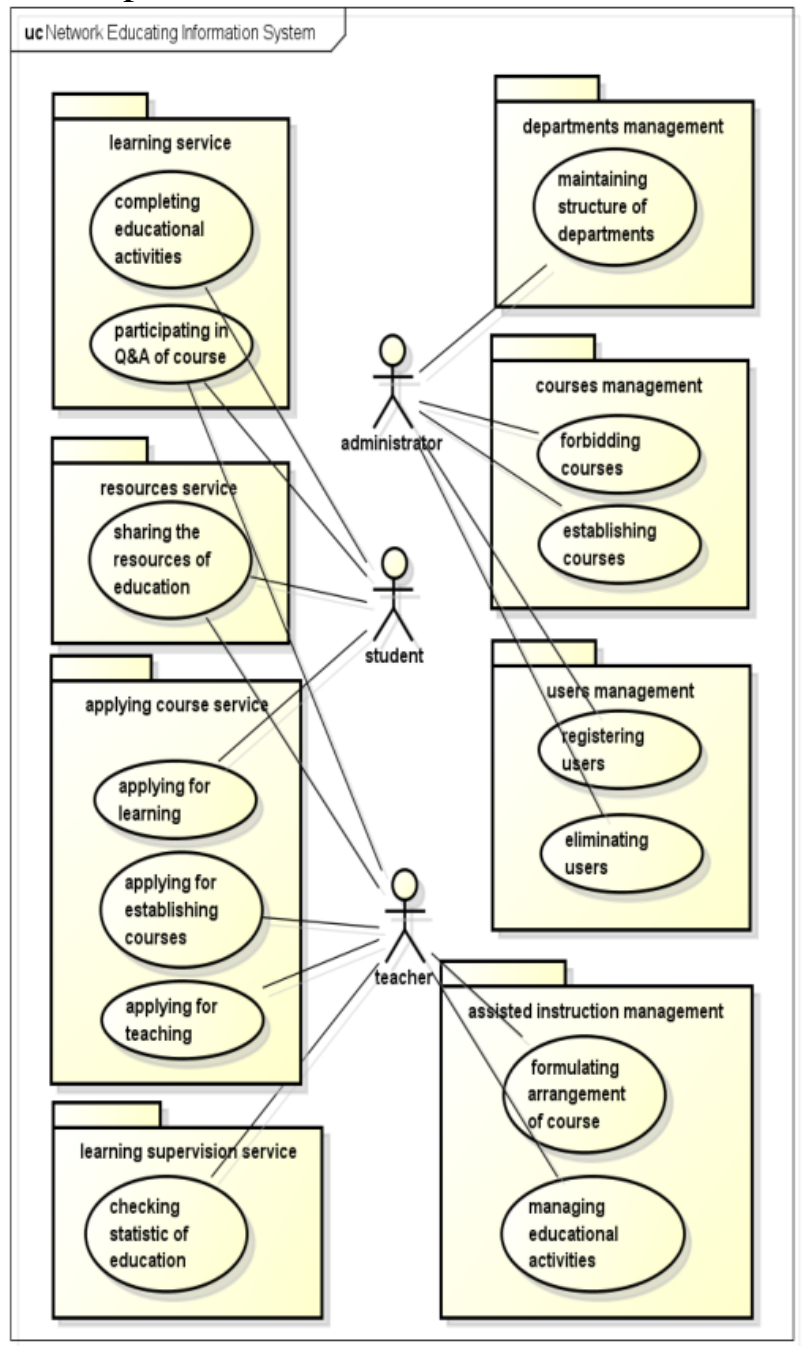

\subsection{Static model}

Figure 3: Use case model

Static model is a view of static structure of the system. Compared with the functions of the system, the structure of static model is unlikely to have change. Static model is developed on basis of use case model. Owing to an array of interactive sequences encapsulated in the inner structure, use cases are described by series of activity diagrams and documents( as is shown in picture 4, an example of applying for establishing courses is cited), so the entities that behave in the inner structure of the system and their static attributes can be captured. All of these entities are integrated in class diagram to describe the whole internal structure of the system, as is described in picture 5 .

\subsection{Dynamic model}

After ensuring static characters of the system, it is indispensable to confirm the information exchange among entities. For every single use case, dynamic model is built by communication diagram. The methods providing or being called by components are depicted in the communication 
diagram. There is the same example of applying for establishing courses, which includes the entities of user, course and applying sheet, as is shown in picture 6. In this communication diagram, it is clear to present the passing massages when the components communicate with each other in the internal system.

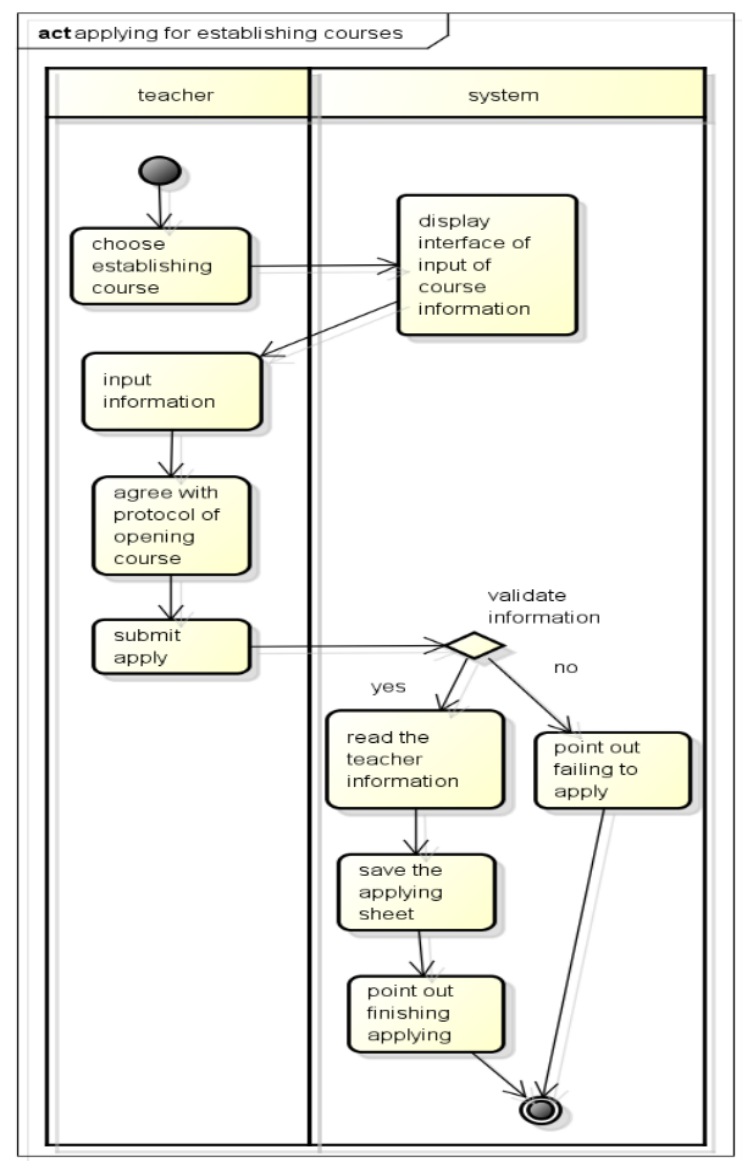

Figure 4: Activity diagram of applying for establishing courses

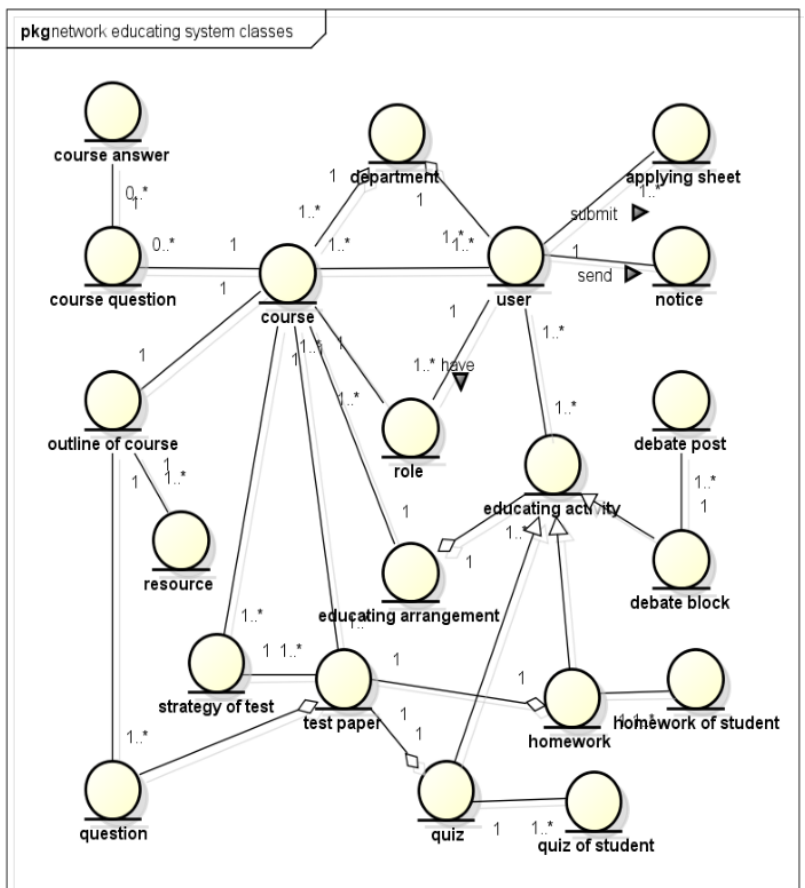

Figure 5: Static model 


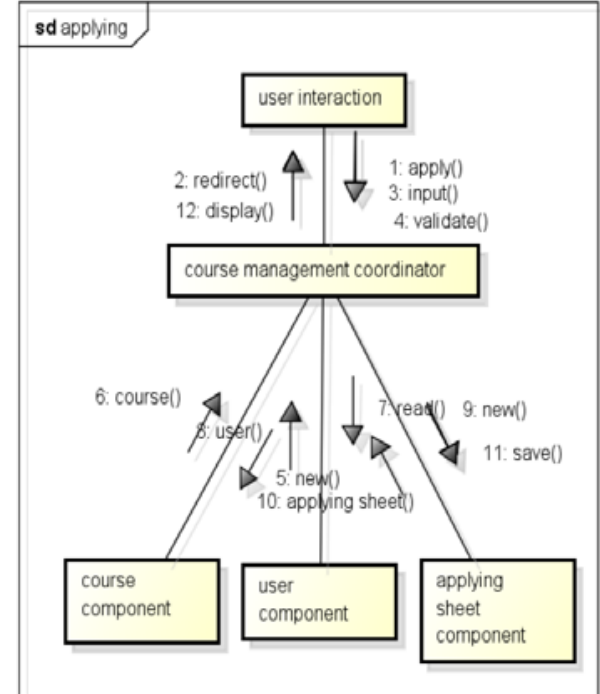

Figure 6: Communication diagram of applying for establishing courses

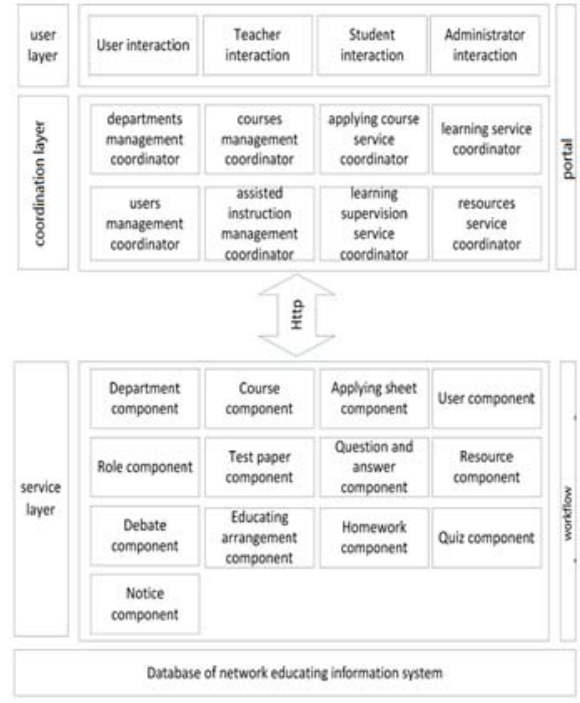

Figure 7: system architecture

\section{System design}

Static and dynamic characters are clearly achieved by utilizing use case model, static model and dynamic model to analyze problem domain. In the procedure of the system design, the system characters are combined with service-oriented architecture to deduce design model of the system. The system is made up of three layers: user layer, coordination layer and service layer. Each component from the same layer is existence to each other in a loosely coupled manner, and the upper layer just depends on lower layer, as is shown in the picture 7. For the system security, by using mechanism of access control based on role, the system assigns the role such as student, teacher, and some extending role to specific user who can have authorities of the role. For designing the course flexibly, functional modules of the course can be pluggable by using portal architecture to provide multiple educating modes. For applying service, the process of the service mainly includes approval process that involves collaborative works among users, so the business of applying is controlled by mechanism of workflow.

\section{Conclusions}

For the shortage of network educating system including single teaching mode and low efficiency of management, the system design uses UML modeling based on use cases driven combining with target decomposition, achieving the design following the analysis, the analysis following the requirement, the requirement following the target. The system has been implemented by portal, workflow, and database technology. The system not only involves traditional educational functions such as learning and management, but also enhances the efficiency of management and gives feedback of the information of educational results positively.

\section{References}

[1] LENG Yue\&XU Chunyu, 2009, Common Misuses of UML Use case tool in software Modeling, China science and technology information, 3, 86.

[2] LIU Xu - yong, 2012, Use Cases Drive Technology Application in Requirements Elicitation Process, computer technology and development, 3, 153.

[3] LIU Zhao\&DONG Ze , 2014, Design and Implementation of a Feed Coal Intelligent Management System Based on UML, computer simulation, 12, 405.

[4] ZHAO Wei-dong, 2013, Design and implemention of fine-grained RBAC model, computer engineering and design, 2,474. 